\title{
O enfoque qualitativo na avaliação do consumo alimentar: fundamentos, aplicaçóes e considerações operacionais
}

I ${ }^{1}$ Maria Lúcia Magalhães Bosi, 2 Shirley Donizete Prado, ${ }^{3}$ Ana Cristina Lindsay, ${ }^{4}$ Márcia Maria Tavares Machado, ${ }^{5}$ Maria Claudia da Veiga Soares Carvalho I

Resumo: Disputas grosso modo infundadas entre defensores dos enfoques qualitativo e quantitativo têm impedido o reconhecimento dos benefícios das aplicaçôes combinadas de ambos os métodos em um mesmo estudo, ou seja, de uma abordagem multidimensional e integrada. Não obstante, em anos recentes, o campo da Nutrição em Saúde Coletiva vem vivenciando um aumento na condução de estudos orientados não apenas pela mensuração, mas pela combinação de métodos qualitativos e quantitativos. Com efeito, o enfoque qualitativo tem muito a contribuir para a investigação do consumo alimentar, dentre vários outros objetos e temáticas, nas quais sobressai a importância do aprofundamento da compreensão da produção subjetiva, expressa em crenças, atitudes e comportamentos. Este artigo resume a natureza, os fundamentos e a utilidade do enfoque qualitativo em pesquisas no âmbito da alimentação e nutrição, esclarecendo como esses métodos têm sido ou podem ser usados para estudar os complexos problemas que se apresentam nesse campo, circunscrevendo a discussão ao âmbito dos estudos sobre consumo alimentar. A integração de ambos os métodos, qualitativo e quantitativo, mediante a complementaridade metodológica, pode minimizar os limites do emprego de cada enfoque de forma isolada.

> Palavras-chave: Pesquisa qualitativa; consumo alimentar; Alimentação e Nutrição; metodologia.

\author{
1 Departamento de Saúde \\ Comunitária. Faculdade de \\ Medicina. Universidade Federal \\ do Ceará. Endereço eletrônico: \\ malubosi@ufc.br \\ ${ }^{2}$ Núcleo de Estudos sobre \\ Cultura e Alimentação \\ (NECTAR). Departamento \\ de Nutrição Social. Instituto \\ de Nutrição. Universidade \\ do Estado do Rio de Janeiro \\ Endereço eletrônico: shirley. \\ prado@yahoo.com.br. \\ ${ }^{3}$ Department of Nutrition \\ Harvard School of Public Health. \\ Endereço eletrônico: alindsay@ \\ hsph.harvard.edu. \\ ${ }^{4}$ Departamento de Saúde \\ Comunitária. Faculdade de \\ Medicina. Universidade Federal \\ do Ceará. Endereço eletrônico: \\ marciamachado@ufc.br.

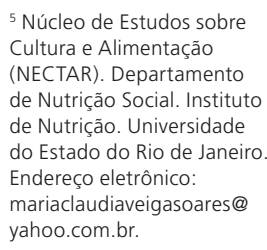

${ }^{5}$ Núcleo de Estudos sobre Cultura e Alimentação (NECTAR). Departamento de Nutrição Social. Instituto de Nutrição. Universidade do Estado do Rio de Janeiro Endereço eletrônico: mariaclaudiaveigasoares@ yahoo.com.br. 
Historicamente, posições por vezes demasiado arraigadas, por parte de defensores dos enfoques qualitativo e quantitativo, têm implicado limitaçôes no que concerne ao reconhecimento dos benefícios e aplicaçôes extensas de ambos os métodos combinados, ou seja, de abordagens multimétodo, no desenho de esforços voltados à geração de conhecimentos e saberes, em saúde, alimentação e nutrição (ALMEIDA; PINTO, 2003; ABUSABHA, WOELFEL, 2003). Nos últimos anos, entretanto, muitas inciativas levadas a cabo nesses domínios têm operacionalizado um incremento expressivo na concepção de estudos com a combinação dos referidos enfoques, ainda que deva ser reconhecida a persistência do predomínio do olhar quantitativo nos estudos de consumo.

Parece haver consenso quanto aos benefícios advindos da combinação de métodos qualitativos e quantitativos no enfrentamento dos complexos problemas de saúde coletiva, incluindo aqueles relacionados à Alimentação e Nutrição. (YACH, 1992; POPE; MAYS, 1995; ABUSABHA, WOELFEL, 2003; COYLE; WILLIAMS, 2000). Neste artigo, embora reconhecendo a inegável contribuição do enfoque quantitativo aos estudos sobre consumo alimentar, nosso foco se volta aos aportes da tradição qualitativa, cujo emprego é ainda minoritário ou mesmo excepcional, haja vista a hegemonia da quantificação em estudos levados a cabo nesse âmbito ${ }^{1}$.

Contudo, a despeito dessa hegemonia e do debate quanti-quali ainda observado nesse domínio, tendências oriundas de olhares mais atentos ao caráter multidimensional dos fenômenos já algum tempo sustentam a complementaridade metodológica como desfecho mais adequado assinalando a utilidade da abordagem qualitativa (ABUSABHA, WOELFEL, 2003; DE VRIES et al., 1992; HUGENTOBLER; ISRAEL, 1992; BUCHANAN, 1992; HELITZERALLEN; KENDALL, 1992; BARBOUR, 1999; GLIK et al., 1987). Metodologias de orientação quantitativa, a despeito de sua inegável utilidade, não dão conta da dimensão subjetiva inerente ao ato alimentar humano. Sendo assim, estratégias metodológicas fundadas nos aportes do enfoque qualitativo podem contribuir expressivamente na compreensão de intervenções, adequação de programas e desenvolvimento de políticas, haja vista que tais ações necessitam considerar o contexto social, econômico e cultural em que esses programas estão inseridos (YACH, 1992; POPE; MAYS, 1995; ABUSABHA, WOELFEL, 2003). 
Embora estudos orientados pela metodologia quantitativa possam oferecer esclarecimentos sobre alguns fatores de risco associados às doenças em determinada população, revela-se igualmente importante investir em outro conjunto articulado de aportes teóricos de vários alcances, de modo a delimitar problemas de investigação sob uma ótica qualitativa. Tal enfoque, demarcado na interface da subjetividade, engendra novas concepções, reconfigurando significados atribuídos a certos conceitos, tais como risco e consumo alimentar, cujo emprego, muitas vezes carece de demarcações mais precisas.

No que concerne ao estudo dos hábitos ou práticas alimentares, conforme já mencionado, é preciso compreender como os indivíduos interpretam o mundo e constroem significados, como se motivam e se relacionam, contextualizando a comida como mediadora de relações, o que implica devolver o caráter complexo aos fenômenos sob estudo. Assim, este ensaio visa a sumarizar os principais fundamentos, bem como alguns aportes e empregos do enfoque qualitativo como ferramenta complementar aos estudos tradicionais voltados ao consumo no campo da Alimentação e Nutrição, ilustrando, ainda, possibilidades de interação dialética e dialógica com as orientações quantitativas.

\section{Consumo alimentar: entre a natureza e a cultura}

Para que se julgue a adequação de uma metodologia ou enfoque ao estudo de um dado objeto - no caso, o consumo alimentar - impõe-se, logo de início, delimitar conceitualmente esse objeto. Noutras palavras, a que nos referimos quando lançamos mão da expressão "consumo alimentar"? Estaremos tratando com um ato fisiológico e inteiramente mensurável, inscrito na natureza, ou com um fenômeno mais complexo que engloba um nível mais imediato, passível de descrição e quantificação, mas, igualmente, nos remete à cultura e à história, demandando, portanto, a compreensão de processos simbólicos e mediações subjetivas que não se submetem à mensuração?

Quando há referência a algo que se ingere para dar conta das necessidades biológicas ligadas à sobrevivência, com efeito, estamos na esfera do natural, da natureza. Sem comer, o ser humano morre; extingue-se a espécie. Contudo, se compreendermos as necessidades humanas como uma questão biológica associada a uma concepção fisiopatológica de saúde, tal como aquela inspirada no modelo biomédico, reproduz-se uma dicotomia entre natureza e cultura; por 
conseguinte, basta-nos quantificar a ingesta, reduzindo sua complexidade à ordem do natural. Nesse sentido, bastante restrito, reduzido a "coisa que serve para o comer humano", encontramos conceituado o alimento na literatura científica atual, conforme ilustram vários textos (PRADO et al., 2011; CARVALHO; LUZ; PRADO, 2011; FRANCO, 1999; BOSI, 1988).

Importante notar que, nessa perspectiva, o conceito de alimento e, por conseguinte consumo alimentar, se apresenta como coisa em si, descontextualizada, desprovida de significado histórico (BOSI, 1988). Desse modo, se opera sua redução a mero veículo de nutrientes interagindo com outro elemento importante - o corpo humano, igualmente tomado como "coisa da natureza". Consumo, nessa perspectiva, é sinônimo de ingestão e o termo alimentar remete, essencialmente, aos nutrientes que integram os alimentos. Entretanto, situar o consumo alimentar como componente da práxis em Alimentação e Nutrição e assumir a avaliação desse consumo como ferramenta de promoção da alimentação saudável implica um trânsito para outros domínios nos quais a dimensão biológica se amplia e se redefine com o aporte das Ciências Humanas e Sociais. Tal trânsito faz com que a concepção, por assim dizer, "naturalizante", se vincule a outros campos como os que insistem em surgir, quando colocamos o alimento no mundo social; mundo em que as pessoas agem, falam, simbolizam e, mais que ingerir alimentos, se engajam em relaçôes mediadas pela comida, ou seja, alimento carregado de significados.

Dessa forma, consumo, muito mais que ingestão, corresponde a uma construção social dialeticamente relacionada com as bases econômicas e históricas que lhe conferem sustentação, bem como com as desigualdades relativas à tomada de decisão sobre o que se produz e ao acesso àquilo que é produzido (PRADO et al., 2011; CARVALHO; LUZ; PRADO, 2011). Só assim o consumo alimentar, que garante a vida em seu sentido biológico, e sem prejuízo desta caracterização, será também compreendido como um ato relacional entre seres humanos, imerso em relaçōes sociais, culturais, políticas, psíquicas, estéticas, dos quais ele representa um dos desfechos.

\section{Aportes do enfoque qualitativo à investigação sobre o consumo alimentar}

Consoante o exposto, ao lado da visão biomédica, e orientado por pressupostos como os que buscamos sumarizar, evidencia-se outro conjunto de esforços, 
mediante os quais se busca aprofundar a análise daquilo que os inquéritos de consumo seguidamente constatam, sem contudo compreender, na acepção epistemológica do termo, o que significa adentrar a produção subjetiva e considerar o consumo alimentar na interface de temas como corpo, alimento, subjetividade, estilos de vida, alteridades.

Para tanto, vários são os aportes do enfoque qualitativo e podemos ilustrá-los dentre outros, como ferramenta a ser utilizada para desenvolver ou aperfeiçoar hipóteses, possibilitando descrições mais refinadas do consumo e compreensão aprofundada dos determinantes sociais de certos comportamentos ou práticas alimentares (DE VRIES et al., 1992; PATTON, 1999). O emprego desse enfoque pode ainda identificar a linguagem mais adequada e significativa a ser utilizada em questionários e outras técnicas de pesquisa inerentes à tradição quantitativa (SEGALL-CORRÊA, 2007).

Além disso, abordagens qualitativas e suas técnicas (por exemplo, entrevistas em profundidade; observações livres ou sistemáticas; grupos focais, fotovoice) são mais adequadas a uma aproximação a objetos complexos que se transformam ao longo da experiência e vão se constituindo de acordo com as percepções dos atores sociais, o repertório e as possibilidades de representação presentes no contexto das relaçôes sociais. Ao investir em abordagens que valorizam a sensibilidade aos símbolos presentes nas interações verbais, nos modos de fazer e de estar, emergem oportunidades singulares para a obtenção de informações novas e inesperadas na situação de pesquisa, tais como percepções, práticas e comportamentos que podem ter como desdobramentos, direta ou indiretamente, reorientaçôes no planejamento e avaliação de intervenções.

Em síntese, cabe reconhecer que, não obstante serem os métodos quantitativos fundamentais para aferir a contribuição de fatores dietéticos no risco para as doenças, dentre várias outras contribuições amplamente documentadas na literatura especializada, a pesquisa qualitativa é fundamental quando se busca ampliar a visão e captar elementos simbólicos e históricos contextuais da realidade, de modo a que se possam desenvolver reflexóes e ações voltadas a mudanças de práticas alimentares. Isso porque perceber elementos variados da realidade é também um modo de organizá-la já que a "realidade" é sempre um universo mais surpreendente do que os modelos tradicionais de ciência podem antecipar. 
Partindo-se dessa perspectiva, depreende-se que a inserção de dimensões qualitativas oportuniza identificar novas terminologias e conceitos no âmbito alimentar-nutricional, assim como na identificação de formas de linguagem mais significativas. Tais elementos ganham relevo uma vez que se desdobram, posteriormente, tanto em instrumentos de pesquisa culturalmente apropriados para grupos populacionais específicos, como em ações voltadas aos problemas identificados, com chances de maior efetividade sobre certos fatores socioculturais e processos subjetivos construídos no cotidiano da existência dos diferentes segmentos sociais e definidores de como as pessoas tomam decisões nas distintas esferas da vida social, dentre elas, a alimentar.

Assim, estudos de consumo alimentar, em seu sentido amplo, se efetivamente visarem a impactar comportamentos e não somente constatá-los, devem englobar certamente a mensuração do que se come, mas dada a multidimensionalidade do fenômeno em tela, necessitam igualmente compreender "como", "porque", "onde", "com quem", "em que ocasiōes" se come - ou não - e a gênese social, cultural e subjetiva das práticas alimentares (PRADO et al., 2011; CARVALHO; LUZ; PRADO, 2011).

Tal assertiva se justifica de forma mais clara quando nos indagamos: para que estudos de consumo alimentar? Supomos que, além do interesse pela reflexão teórica ou conceitual dos grupos de pesquisa e profissionais do campo, tais estudos devam, sobretudo, guiar intervençōes. Podemos ainda afirmar que o investimento em estratégias qualitativas responde a uma demanda do campo da alimentação e nutrição, haja vista o ato alimentar assumir uma dimensão vital na pesquisa afiliada a uma concepção de saúde ampliada. Desse modo, a despeito da importância, não basta nos determos no cálculo de probabilidades de adoecimento, ainda que verificados e validados mediante procedimentos sofisticados. Há também que estar atento ao que o homem constrói como elemento de seu bem estar, ou como projeto de felicidade (AYRES, 2004).

Nesse sentido, conhecer um objeto como o que aqui focalizamos demanda valorizar relações sócio-simbólicas para, posteriormente, operacionalizar os cruzamentos com teorias auxiliares oriundas das diversas tradiçôes na construção das concepções, seja de risco ou de saúde. Sendo assim, questôes bem mais complexas e menos visíveis nos desafiam e o enfoque qualitativo nos ajuda a esclarecê-las, subsidiando as ações no setor. 
Apontada a natureza, bem como os principais fundamentos e alguns aportes desse enfoque ao campo dos estudos sobre consumo alimentar, finalizaremos este artigo com observações direcionadas a alguns elementos centrais concernentes à dimensão técnico-operacional dessa vertente que nos parece oportuno comentar.

\section{Acerca das técnicas e outros procedimentos}

No escopo da tradição qualitativa, uma primeira questão a assinalar refere-se à postura observada com certa frequência, que considera uma investigação como sendo "qualitativa" pelo fato de empregar alguma(s) técnica(s) "qualitativa(s)". Trata-se de um equívoco, com consequências importantes, resultante da confusão entre método e técnica. Consoante Bosi e Mercado (2006):

A redução de um plano (método) ao outro (técnica), possibilita [...] que o emprego de uma técnica substitua a necessária presença de uma 'postura qualitativa', ou seja, um fundamento epistemológico a partir do qual as técnicas façam sentido - um método - e do qual as mesmas se originem, sejam elas grupos focais, entrevista em profundidade, observação participante ou qualquer outra relacionada com essa tradição. [...] Em síntese, uma investigação não deve ser considerada qualitativa ou quantitativa simplesmente pelo fato de empregar uma determinada técnica. Ao contrário, é imprescindível levar em conta: 1) a pergunta ou objeto da investigação 2) uma determinada postura teórica ou epistemológica e 3) a correspondente estratégia para a obtenção das informações e sua análise.

No que diz respeito ao objeto de investigação, estudos com uma orientação qualitativa podem ter como objeto certos tipos de comportamento, ações ou práticas, mas o material serásempre aquele cuja expressão não se submeteà linguagem numérica; ou seja, tal enfoque se aplica a perguntas (objetos) que demandam compreensão.

Quanto ao segundo aspecto assinalado na citação acima, estudos qualitativos não se caracterizam apenas por empregar determinadas técnicas, nem por fazer perguntas diferentes das convencionais; trata-se de um enfoque com diversas implicações e a relação entre pergunta e técnicas de pesquisa dista de ser imediata e unilinear (BOSI; MERCADO, 2006). Além disso, cabe mencionar como "marca de origem" que as posturas teóricas e as terminologias empregadas nesse enfoque remetem a múltiplas concepçōes, tendências, escolas, tradições e posturas existentes nesse campo (DENZIN; LINCOLN, 2000). Acrescente-se a coexistência de múltiplos desenhos ou estratégias de investigação que podem ser empregadas no campo da investigação qualitativa e, mais especificamente, na avaliação do consumo alimentar de distintos grupos populacionais. 
Cada estratégia ou modelo tem seus pontos fortes e suas debilidades. Por tal motivo, e tendo presentes os temas e interesses dos que, como nós, se dedicam à pesquisa qualitativa, cada vez mais se aceita a proposta da triangulação metodológica (DENZIN, 1970) no interior do mesmo paradigma - como uma estratégia complementar de trabalho. Em termos gerais, a triangulação metodológica dá conta, no campo específico da pesquisa qualitativa, do uso de diversas teorias, estratégias, métodos, procedimentos ou informações/dados em torno do mesmo objeto ou fenômeno sob estudo. Tal proposição conflui para propostas que sustentam a combinação desses desenhos com aqueles de natureza quantitativa, tal como logo de início salientamos nesta discussão.

A integração dos dois enfoques pode fornecer dados e informaçōes referidos a diferentes planos ou dimensões de análise, respondendo a perguntas sob múltiplas perspectivas, enriquecendo nosso conhecimento sobre a alimentação e a nutrição, suas relações com a saúde. Superando disputas e outras posturas equivocadas como as que advogam por uma harmonia paradigmática, tal integração, reconhecendo as distinçôes entre os enfoques, mas também o valor de cada tradição, viabiliza a descrição, mas associando-a a uma compreensão mais abrangente e refinada das motivaçóes e comportamentos subjacentes às complexas questóes que desafiam o campo da saúde. Justificam-se, portanto, esforços que promovam o desenho de estudos capazes de subsidiar a implementação de políticas e programas inovadores e exitosos ante o cenário que nos desafia no âmbito da alimentação e nutrição humanas.

\section{Referências}

ABUSABHA, R.; WOELFEL, M.L. Qualitative vs quantitative methods: two opposites that make a perfect match. JAm Diet Assoc., v. 103, n. 5, p. 566-9, May 2003.

ALMEIDA, J.F.; PINTO, J.M. Da teoria à investigação empírica. Problemas metodológicos gerais. In: SILVA, A.S.; PINTO, J.M. (Org.). Metodologia das Ciências Sociais. Porto: Afrontamento, 2003. p. 55-78.

AYRES, J.R.C. Uma concepção hermenêutica de saúde. Physis: revista de saúde coletiva, v. 17, p. 43-62, 2007.

BARBOUR, R.S. The case for combining qualitative and quantitative approaches in health services research. J Health Serv Res Policy, v. 4, n. 1, p. 39-43, Jan 1999.

BOSI, M.L.M.; MERCADO, F.J. (Org.). Pesquisa qualitativa de serviços de saúde. Petrópolis: Vozes; 2006.

BOSI, M.L.M. A face oculta da Nutrição: ciência e ideologia. Rio de Janeiro: Espaço e Tempo, 1988. 
BUCHANAN, D.R. An uneasy alliance: combining qualitative and quantitative research methods. Health Educ Q., v. 19, n. 1, p. 117-35, Spring 1992.

CARVALHO, M.C.V.S.; LUZ, M.T.; PRADO, S.D. Comer, nutrir, alimentar: categorias analíticas instrumentais no campo da pesquisa científica. Ciência \& Saúde Coletiva, v 16, n 1, p. 155-163, 2011.

COYLE, J.; WILLIAMS, B. An exploration of the epistemological intricacies of using qualitative data to develop a quantitative measure of user views of health care. J Adv Nurs., v. 31, n. 5, p. 2000 May;31(5):1235-43.

DE VRIES, H. et al. The utilization of qualitative and quantitative data for health education program planning, implementation, and evaluation: a spiral approach. Health Educ Q., v. 19, n. 1, p. 101-15, Spring 1992.

DENZIN, N. The research act. Chicago: Aldine, 1970.

DENZIN, NK; LINCOLN, Y. Handbook of Qualitative Research. Thousand Oaks: Sage, 2000.

FRANCO, G. Tabela de composição química dos alimentos. São Paulo: Atheneu, 1999.

GLIK, D. et al. Focus group methods for formative research in child survival: an ivoirian example. Int Q Community Health Educ., v. 8, n. 4, p. 297-316, Jan 1987.

HELITZER-ALLEN, D.L.; KENDALL, C. Explaining differences between qualitative and quantitative data: a study of chemoprophylaxis during pregnancy. Health Educ Q., v, 19, n. 1, p. 41-54, Spring 1992.

HUGENTOBLER, M.K.; ISRAEL, B.A.; SCHURMAN, S.J. An action research approach to workplace health: integrating methods. Health Educ Q., v. 19, n. 1, p. 55-76, Spring 1992. PATTON, M.Q. Enhancing the quality and credibility of qualitative analysis. Health Serv Res., v. 34, n. 5 Pt 2, p. 1189-208, Dec 1999.

POPE, C.; MAYS, N. Reaching the parts other methods cannot reach: an introduction to qualitative methods in health and health services research. BMJ., v. 311, n. 6.996, p, 42-5, Jul 1, 1995.

PRADO, S.D. et al. A pesquisa sobre Alimentação no Brasil: sustentando a autonomia do campo alimentação e nutrição. Ciência \& Saúde Coletiva, v. 16, n. 1, p. 107-120, 2011.

SEGALL-CORRÊA, A. M. Insegurança Alimentar medida a partir da percepção das pessoas. Estudos Avançados, v. 21, p. 143/60-154, 2007.

YACH, D. The use and value of qualitative methods in health research in developing countries. Soc Sci Med., v. 35, n. 4, p. 603-12, Aug 1992.

\section{Nota}

${ }^{1}$ Nossos agradecimentos pelo apoio financeiro recebido do CNPq através do processo 473853/20078 e da FAPERJ por meio do processo E-26/110.393/2008. 


\section{The qualitative approach in the evaluation of food consumption: fundamentals, applications and operational considerations}

Unfounded disputes between advocates of qualitative and quantitative approaches have hindered the recognition of the benefits of combined application of both methods in the same study, ie, a multidimensional and integrated approach. Nevertheless, in recent years, the field of Nutrition in Public Health has experienced an increase in conducting studies guided not only by measurement, but by the combination of qualitative and quantitative methods. Indeed, the qualitative approach has much to contribute to research in food consumption, among many other objects and themes, in which stands the importance of deepening the understanding of subjective production, expressed in beliefs, attitudes and behaviors. This paper summarizes the nature, rationale and usefulness of the qualitative research approach in Food and Nutrition, explaining how these methods have been or may be used to study the complex problems that arise in this field, limiting the discussion to the scope of the studies on food consumption. The integration of both methods, qualitative and quantitative, by the methodological complementarity, can minimize the limits of use of each approach in isolation.

> Key words: qualitative research; food consumption; Food and Nutrition; methodology. 